\section{ORIGINAL RESEARCH}

\author{
A.C. Flint \\ S.P. Cullen
}

B.S. Faigeles

V.A. Rao

\title{
Predicting Long-Term Outcome after Endovascular Stroke Treatment: The Totaled Health Risks in Vascular Events Score
}

\begin{abstract}
BACKGROUND AND PURPOSE: Endovascular treatments are being increasingly used in acute ischemic stroke, and better tools are needed to determine which patients may benefit most from these techniques. We hypothesized that specific chronic diseases can be used, along with age and stroke severity, to predict endovascular stroke treatment outcomes.
\end{abstract}

MATERIALS AND METHODS: Data from 2 single-arm trials of a thrombectomy device, $\mathrm{MERCl}$ and Multi $\mathrm{MERCl}$, were pooled for analysis. A predictive score was developed by using the independent contribution of variables in multivariable analysis.

RESULTS: HTN, DM, and AFib were found to predict outcomes. These 3 conditions contribute equally to a CDS that predicts outcomes independent of other predictor variables, including age, stroke severity, and vessel recanalization. A 10-level predictive score, the THRIVE score, which incorporates age, stroke severity, and the CDS, was developed. The THRIVE score strongly predicts outcome and mortality at 90 days.

CONCLUSIONS: Specific chronic diseases influence poststroke outcomes among patients undergoing endovascular stroke treatment, independent of other predictors of outcome. The THRIVE score reflects the contributions of chronic disease, age, and stroke severity and strongly predicts endovascular stroke treatment outcomes.

\begin{abstract}
ABBREVIATIONS: AFib = history of atrial fibrillation; ASPECTS = Alberta Stroke Program Early CT Score; $C A D=$ history of coronary artery disease; $C D S=$ chronic disease scale; CHF = history of congestive heart failure; $\mathrm{Chol}=$ history of hypercholesterolemia; $\mathrm{Cl}=$ confidence interval; $\mathrm{DM}=$ history of diabetes mellitus; GLM = generalized linear model; HTN = history of hypertension; $\mathrm{MERCl}=$ Mechanical Embolus Removal in Cerebral Ischemia; $\mathrm{mRS}=$ modified Rankin Scale; Multi $\mathrm{MERCl}=$ Multi Mechanical Embolus Removal in Cerebral Ischemia; NIHSS = National Institutes of Health Stroke Scale; OR = odds ratio; PROACT = Prolyse in Acute Cerebral Thromboembolism; Pseudo- $R^{2}=$ pseudo R-squared; ROC AUC = receiver-operating characteristic area under the curve; RR = risk ratio; THRIVE = Totaled Health Risks in Vascular Events score; Tob = history of active tobacco smoking; tPA = tissue plasminogen activator
\end{abstract}

E ndovascular stroke treatments are being increasingly used for acute ischemic stroke. Although the randomized controlled trial PROACT II $^{1}$ stands as a proof of the principle that vessel recanalization by endovascular stroke treatment should be beneficial, current methods for endovascular stroke treatment (mechanical thrombectomy and intra-arterial tPA treatment) remain untested at the randomized clinical trial level. The single-arm MERCI and Multi MERCI trials showed the independent association of vessel recanalization with improved clinical outcomes, across all vessel targets and controlling for patient factors known to influence poststroke outcome such as age and stroke severity. ${ }^{2-6}$ When MERCI and Multi MERCI patients who would have been eligible for PROACT II were compared with the treatment arm of PROACT II, the MERCI and Multi MERCI outcomes compared favorably. ${ }^{7}$

Received November 2, 2009; accepted after revision December 17.

From the Departments of Neurocritical Care and Stroke (A.C.F., B.S.F., V.A.R.) and Interventional Neuroradiology (S.P.C.), Regional Neuroscience Program, Kaiser Permanente Northern California, Redwood City, California.

Please address correspondence to Alexander C. Flint, MD, PhD, Kaiser Permanente, 1150 Veterans Blvd., Redwood City, CA 94063; e-mail: alexander.c.flint@kp.org

Indicates article with supplemental on-line table.

DOI 10.3174/ajnr.A2050
There is a need to better define the population of patients who might benefit most from endovascular stroke treatment. Decisions regarding patient eligibility for endovascular treatment are complex and often influenced by patient age and the presence of medical comorbidities. With this in mind, we used data from the MERCI and Multi MERCI trials to test whether the burden of specific chronic diseases can be used, along with age and stroke severity, to build a score that predicts outcomes among patients undergoing endovascular stroke treatment.

\section{Materials and Methods}

Data for our analyses were derived from 2 trials of the Merci retriever (Concentric Medical, Mountain View, California): the MERCI trial and the Multi MERCI trial. The details of the trial structure for MERCI and Multi MERCI were very similar and have been described in detail elsewhere. ${ }^{2,3}$

\section{Patients and Techniques}

Both MERCI and Multi MERCI were single-arm multicenter trials. ${ }^{2,3}$ The major difference between MERCI and Multi MERCI was that Multi MERCI allowed inclusion of both intravenous tPA-ineligible and intravenous tPA - treated patients. ${ }^{2}$ Details of patient enrollment in MERCI and Multi MERCI, including inclusion and exclusion criteria, have been previously described., ${ }^{2,3}$ Details of the devices and 


\begin{tabular}{|c|c|c|c|c|c|c|}
\hline Condition & Poor Outcome (mRS 3-6) & Good Outcome (mRS 0-2) & Total (mRS 0-6) & $\mathrm{OR}$ & $P$ Value & Adjusted $O \mathrm{R}^{\mathrm{b}}$ \\
\hline$\overline{\mathrm{HTN}}$ & $77.4 \%(151 / 195)$ & $60.6 \%(57 / 94)$ & $72.0 \%(208 / 289)$ & 0.45 & $<.01$ & 0.56 \\
\hline AFib & $46.6 \%(90 / 193)$ & $31.9 \%(30 / 94)$ & $41.8 \%(120 / 287)$ & 0.54 & .02 & 0.53 \\
\hline DM & $23.8 \%(46 / 193)$ & $12.8 \%(12 / 94)$ & $20.2 \%(58 / 287)$ & 0.47 & .03 & 0.61 \\
\hline CAD & $45.0 \%(85 / 189)$ & $34.0 \%(32 / 94)$ & $41.3 \%(117 / 283)$ & 0.63 & .10 & $\mathrm{n} / \mathrm{a}$ \\
\hline CHF & $21.6 \%(41 / 190)$ & $14.9 \%(14 / 94)$ & $19.4 \%(55 / 284)$ & 0.64 & .20 & $\mathrm{n} / \mathrm{a}$ \\
\hline Tob & $21.6 \%(39 / 181)$ & $23.9 \%(21 / 88)$ & $22.3 \%(60 / 269)$ & 1.14 & .76 & $\mathrm{n} / \mathrm{a}$ \\
\hline Chol & $33.9 \%(59 / 174)$ & $35.2 \%(32 / 91)$ & $34.3 \%(91 / 265)$ & 1.06 & .89 & $\mathrm{n} / \mathrm{a}$ \\
\hline
\end{tabular}

${ }^{a}$ Data are presented as percentages in each outcome category for a given diagnosis, followed by the number of patients with the diagnosis in this category over the total number of patients in this category.

${ }^{\mathrm{b}}$ OR for good outcome in multivariable logistic regression, including the variables HTN, AFib, and DM.

procedures used in MERCI and Multi MERCI have been previously published. ${ }^{2,3}$ Successful recanalization was defined as achieving a Thrombolysis in Myocardial Infarction ${ }^{8} 2$ or 3 flow in all treatable vessels. Intra-arterial thrombolytics were allowed in cases of treatment failure with the device or to treat distal embolus not accessible to the device. Angioplasty or stent placement or both were not allowed. Baseline clinical variables included 7 chronic health variables: HTN, AFib, DM, CAD, CHF, current tobacco use, and Chol. Primary outcomes in the original MERCI and Multi MERCI trials were vessel recanalization and device-related complications. Secondary outcomes in the original MERCI and Multi MERCI trials included the mRS and NIHSS scores at 30 and 90 days. The prespecified definition of good neurologic outcome was mRS 0-2.

\section{Statistical Analysis}

Categoric data in contingency tables were analyzed by the Fisher exact test. For contingency tables with an ordinal predictor variable and a dichotomous outcome variable, the Mantel-Haenszel $\chi^{2}$ test for trend of odds was used. Univariable analyses were performed for each of 7 chronic disease states to examine the association with good outcome (mRS 0-2) at 90 days. Multivariable models were built by using logistic regression and binomial GLM for dichotomous outcomes (eg, good-versus-poor outcome) and ordinal logistic regression for ordinal outcomes (mRS 0 through 6). All multivariable models in the present analysis used simultaneous variable entry. For both the CDS and the THRIVE score (see text for definitions), the relative weighting of individual components of each was determined from the relative change of the OR of the variables from unity in the appropriate multivariable model. Cut points used for the NIHSS were chosen to produce reasonable categories of mild $(\leq 10)$, moderate (11-20), and severe $(\geq 21)$ strokes for the patient population with large artery occlusions included in MERCI and Multi MERCI, with rounding of the cut point to the nearest decade of NIHSS to facilitate easy recall. Cutpoints for age ( $\leq 59,60-79, \geq 80$ years) were chosen to approximate the tertiles of age in the dataset, with rounding to the nearest decade of age to facilitate easy recall. To evaluate whether addition of the CDS improves the multivariable model of good outcome, we used Pseudo- $R^{2}$ and ROC AUC to compare logistic regression models with and without the CDS. All statistical analyses were performed by using STATA/SE, Version 10.0 (StataCorp, College Station, Texas).

The MERCI and Multi MERCI trials received institutional review board approvals from the institutions involved in the conduct of these 2 clinical trials. No protected health information was transmitted from Concentric Medical to the authors of the present study.

\section{Results}

To explore the relationship between chronic diseases and clinical outcomes among patients undergoing endovascular

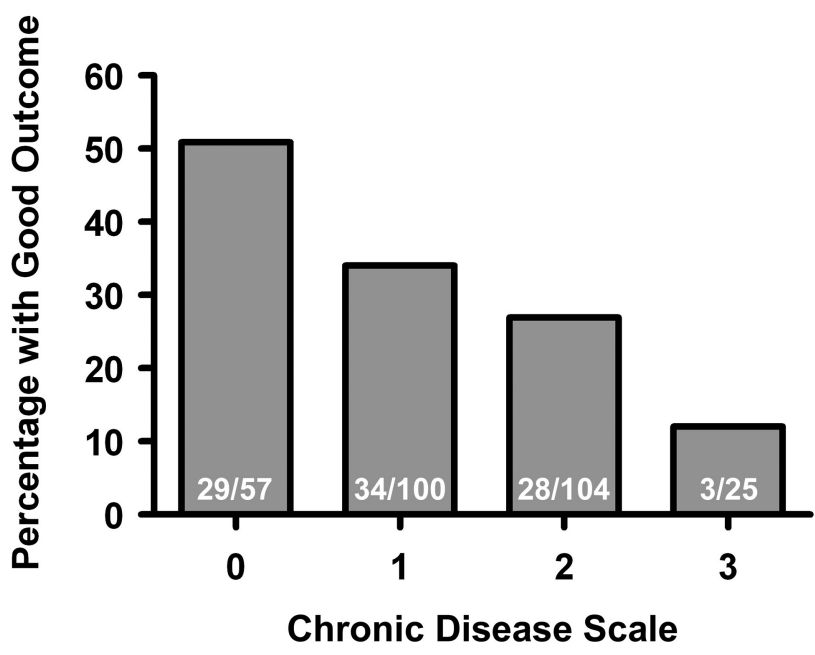

Fig 1. Association of CDS with outcome. A progressively lower percentage of patients with good outcomes (mRS $0-2$ at 90 days) is seen with each increased level of the CDS.

stroke treatment, we combined the 2 largest published datasets, the MERCI and Multi MERCI trials, ${ }^{2-4}$ with a total of 305 patients. We first performed univariable analyses exploring the relationship between 7 chronic health conditions and the odds of good outcome (mRS $0-2$ at 90 days poststroke). Table 1 shows the percentage of patients with each of these 7 chronic health conditions according to clinical outcome at 90 days (poor outcome of mRS 3-6 versus good outcome of mRS 0-2) and the corresponding ORs for each condition. Of the 7 health conditions, only HTN, DM, and AFib had a statistically significant association with outcome at 90 days. Each of these 3 chronic health conditions had an OR for good outcome of approximately 0.5 in univariable analysis.

When these 3 conditions (HTN, DM, and AFib) were examined in multivariable logistic regression modeling good outcome at 90 days, the point estimates for the ORs were not substantially altered (Table 1). Because each condition is associated with a reduction in the point estimate of the odds of good outcome by approximately half, we created a CDS in which the presence of each chronic health condition is weighted equally on a 0 - to 3 -point scale. Each increased level of CDS is associated with a significantly decreased chance of good outcome (Fig 1). Good outcomes were seen in the following percentages of patients at each level of the CDS: $50.9 \%$ at 0 points, $34.0 \%$ at 1 point, $26.9 \%$ at 2 points, and $12.0 \%$ at 3 points $\left(P<.001\right.$, Mantel-Haenszel $\chi^{2}$ test $)$. Multivariable logistic regression with each level of the CDS modeling good 


\begin{tabular}{lccr}
\hline \multicolumn{4}{l}{$\begin{array}{l}\text { Table 2: Multivariable logistic regression: association of age, } \\
\text { NIHSS, recanalization, and CDS } \text { with good outcome }\end{array}$} \\
\hline \multicolumn{1}{l}{ Variable } & OR & $95 \%$ Cl & $P$ Value \\
\hline Age & 0.975 & $0.955-0.996$ & .019 \\
NIHSS score & 0.890 & $0.842-0.940$ & $<.001$ \\
Recanalization & 19.73 & $7.61-51.23$ & $<.001$ \\
CDS & 0.665 & $0.456-0.970$ & .034 \\
\hline
\end{tabular}

outcome confirms that increasing levels of the CDS are independently associated with reduced odds of a good outcome (on-line Table). Ordinal logistic regression modeling the full range of mRS (0-6) shows that each increased level of the CDS is associated with an OR of 1.59 for each increased level of the mRS $(P<.001)$.

Because other studies have found that hyperglycemia on initial glucose measurement at acute stroke presentation is associated with poor outcome after endovascular stroke treatment, ${ }^{9}$ we also examined whether admission hyperglycemia yielded different results from a history of DM. When DM was replaced in the above analyses with admission hyperglycemia (glucose $>150 \mathrm{mg} / \mathrm{dL}^{9}$ ) the results were not substantially altered (data not shown).

We next asked whether the CDS predicts clinical outcome independent of other factors known to be associated with outcome after endovascular stroke treatment such as age, NIHSS at presentation, and vessel recanalization..$^{2-4,10}$ A multivariable logistic regression model of good outcome that includes age, NIHSS, vessel recanalization, and the CDS shows that each of these variables is independently associated with clinical outcome at 90 days (Table 2). Each level of the CDS is associated with a reduction in the odds of a good outcome by approximately one-third, after controlling for the effects of age, NIHSS, and vessel recanalization. Finally, we investigated whether adding information on chronic diseases improved prediction of good outcome, net of the usual predictors (age and NIHSS). Table 3 includes 2 measures of fit for the 2 models as well as the contributions of each of the 3 predictor variables (age, NIHSS, and CDS). When CDS is added, model fit improves by both measures: the Pseudo- $R^{2}$ increases from 0.0804 to 0.0928 and the ROC AUC increases from 0.683 to 0.709 .

We then constructed a broader score, the THRIVE score, to predict outcomes among patients undergoing endovascular stroke treatment. To create a simple-to-use scoring system, we trichotomized the variables age and NIHSS (Table 4). When trichotomized age ( $\leq 59,60-79$, and $\geq 80$ years), trichotomized NIHSS ( $\leq 10,11-20$, and $\geq 21$ ), and the CDS are entered into a logistic regression model of good outcome at 90 days, the OR point estimates reflect a relative 2-fold impact of each level of the trichotomized NIHSS $(\mathrm{OR}=0.388)$ and a relative 1-fold impact of each level of trichotomized age $(\mathrm{OR}=0.724)$ and the $\mathrm{CDS}(\mathrm{OR}=0.683)$. Negative binomial regression to yield independent relative risks for each variable in association with good outcome confirmed the relative impact of these 3 variables (trichotomized NIHSS RR $=0.657$, trichotomized age $\mathrm{RR}=0.835$, and CDS RR $=0.822$ ). The THRIVE score was, therefore, constructed to assign 2 points for each level of the trichotomized NIHSS, 1 point for each level of trichotomized age and 1 point for each level of the CDS (Table 4).
The THRIVE score strongly predicts outcomes among patients undergoing endovascular stroke treatment (Fig 2). Patients with a low THRIVE score of $0-2$ had good outcomes in $64.7 \%$ of cases, patients with a moderate THRIVE score of 3-5 had good outcomes in $43.5 \%$ of cases, and patients with a high THRIVE score of 6-9 had good outcomes in $10.6 \%$ of cases (Fig 2A; $P<.001$, Mantel-Haenszel $\chi^{2}$ test). Patients with a low THRIVE score of $0-2$ had a mortality rate of $5.9 \%$ by 90 days, patients with a moderate THRIVE score of 3-5 had a mortality rate of $30.1 \%$ by 90 days, and patients with a high THRIVE score of $6-9$ had a mortality rate of $56.4 \%$ by 90 days (Fig 2B; $P<.001$, Mantel-Haenszel $\chi^{2}$ test). The strong association of THRIVE score with the range of clinical outcomes measured by the mRS is shown in Fig $2 C$, in which the range of mRS scores is shown for 3 strata of the THRIVE score $(0-2$, $3-5$, and $6-9)$. In ordinal logistic regression modeling the full range of the mRS (0-6), each increased level of the THRIVE score is associated with an OR of 1.47 for each increased level of the mRS $(P<.001)$.

The independent association of THRIVE score and vessel recanalization with good outcome was seen in multivariable logistic regression, in which the OR for good outcome for each level of the THRIVE score was 0.608 and the OR for good outcome for successful recanalization was $20.99(P<.001$ for both variables). In ordinal logistic regression modeling the full range of the mRS with both the THRIVE score and recanalization in the model, the OR for each level of increased mRS for each level of increased THRIVE score was 1.518, while the OR for each level of increased $\mathrm{mRS}$ for successful recanalization was 0.182 .

The THRIVE score is associated with outcome in patients who had successful vessel recanalization as well as those who did not have successful recanalization. Among patients with successful recanalization, those with a low THRIVE score of $0-2$ had good outcomes in $82.6 \%$ of cases, those with a moderate THRIVE score of 3-5 had good outcomes in $60.0 \%$ of cases, and those with a high THRIVE score of 6-9 had a good outcome in $18.5 \%$ of cases $\left(P<.001\right.$, Mantel-Haenszel $\chi^{2}$ test). Among patients without successful recanalization, those with a low THRIVE score of $0-2$ had good outcomes in $27.3 \%$ of cases, those with a moderate THRIVE score of 3-5 had good outcomes in $6.9 \%$ of cases, and those with a high THRIVE score of 6-9 had a good outcome in $0 \%$ of cases $(P=.001$, Mantel-Haenszel $\chi^{2}$ test). Among patients with successful recanalization, those with a low THRIVE score of $0-2$ had a mortality rate of $0 \%$ by 90 days, those with a moderate THRIVE score of $3-5$ had a mortality rate of $19.4 \%$ by 90 days, and those with a high THRIVE score of 6-9 had a mortality rate of $48.5 \%$ by 90 days $\left(P<.001\right.$, Mantel-Haenszel $\chi^{2}$ test). Among patients without successful recanalization, those with a low THRIVE score of $0-2$ had a mortality rate of $18.2 \%$ by 90 days, those with a moderate THRIVE score of 3-5 had a mortality rate of $53.3 \%$ by 90 days, and those with a high THRIVE score of $6-9$ had a mortality rate of $67.4 \%$ by 90 days $(P=$ .004 , Mantel-Haenszel $\chi^{2}$ test).

\section{Discussion}

Our analyses show that among patients undergoing endovascular stroke treatment in the MERCI and Multi MERCI trials, the THRIVE score strongly predicts 90 -day clinical outcomes. 


\begin{tabular}{|c|c|c|c|c|c|}
\hline \multicolumn{2}{|c|}{ Model 1} & \multirow{2}{*}{$\begin{array}{l}\text { Pseudo- } R^{2}=0.0804 ; \\
\text { ROC AUC }=0.683 \\
P \text { Value }\end{array}$} & \multicolumn{2}{|c|}{ Model 2} & \multirow{2}{*}{$\begin{array}{c}\text { Pseudo- } R^{2}=0.0928 \\
\text { ROC AUC }=0.709 \\
P \text { Value }\end{array}$} \\
\hline Variable & $\mathrm{OR}$ & & Variable & $\mathrm{OR}$ & \\
\hline Age (3 levels) ${ }^{a}$ & 0.609 & .007 & Age (3 levels) & 0.724 & .097 \\
\hline NIHSS (3 levels) ${ }^{b}$ & 0.353 & $<.001$ & $\begin{array}{l}\text { NIHSS (3 levels) } \\
\text { CDS }^{\mathrm{b}}\end{array}$ & $\begin{array}{l}0.388 \\
0.683\end{array}$ & $\begin{array}{r}<.001 \\
.021\end{array}$ \\
\hline
\end{tabular}

age trichotomized as shown in Table 4.
${ }^{\mathrm{b}}$ NIHSS trichotomized as shown in Table 4

c See Table 4.

\begin{tabular}{lc}
\hline \multicolumn{1}{l}{ Table 4: The totaled health risks in vascular events (THRIVE) score } \\
\hline \multicolumn{2}{l}{ Points } \\
\hline NIHSS & 0 \\
$\leq 10$ & 2 \\
$11-20$ & 4 \\
$\geq 21$ & \\
Age & 0 \\
$\leq 59$ & 1 \\
$60-79$ & 2 \\
$\geq 80$ & \\
CDS (1 point each for HTN, DM, AFib) & 0 \\
0 & 1 \\
1 & 2 \\
2 & 3 \\
3 & THRIVE score $=$ \\
\end{tabular}

If validated in future analyses, the THRIVE score may prove useful in improving patient selection in clinical trials and in clinical practice.

The CDS, an evenly weighted 3-point score encompassing a history of HTN, DM, and Afib, is associated with 90-day outcomes, independent of factors such as age, stroke severity, and successful vessel recanalization. When the CDS is added to a logistic regression model containing age and NIHSS, the relationship between age and good outcome is weakened (Table 3); this finding suggests that the impact of age on good outcome in this population might be partially accounted for by the accumulation of chronic disease burden as a function of age. Moreover, the net change in the odds of a good outcome as one moves from 1 level of the CDS to the next is about the same as the change associated with moving from 1 age category to the next. In other words, knowing about comorbidities may significantly improve the clinician's ability to predict how well a patient will do after endovascular stroke treatment. It may, therefore, be wise to avoid considering age alone, without factoring in chronic disease burden, in making decisions regarding endovascular stroke treatment. The general theme of a scoring system for burden of chronic health conditions may be of relevance to predict outcomes in other situations, such as ischemic stroke in general or in other diagnoses. However, the CDS developed here should be considered specific to prediction of outcome in the endovascular stroke patient population until it is further explored in other contexts.

Many modifiable and nonmodifiable factors measurable at admission have been shown to influence clinical outcomes following acute ischemic stroke. ${ }^{10}$ Modifiable predictors of outcome include vessel recanalization, blood pressure, glucose level, patient temperature, and care in an organized stroke unit. ${ }^{10}$ Nonmodifiable predictors of outcome include age, initial stroke severity, stroke territory and subtype, imaging findings, and medical comorbidities. ${ }^{10}$ The association of the THRIVE score with outcome appears to be strong, but it must be interpreted in the broader context of this long list of predictive factors.

The strengths of our analyses include the use of clinical trial data with strict inclusion criteria and adjudication of patient data and end points, the long-term outcomes of mRS and mortality at 90 days, and the generation of a clinical predictive score with 10 levels, which yields a level of granularity that may prove useful if the THRIVE score is adopted into research or clinical use. The generation of the scoring systems by multivariable logistic regression and weighting by relative ORs derived from a reasonable-sized cohort may also make these scores more robust when applied to other datasets.

The weaknesses of our analyses include the lack of imaging data and the use of data from trials of only 1 type of endovascular stroke treatment (the Merci retriever). The clinical comorbidities recorded in MERCI and Multi MERCI were limited to the variables explored in the present analysis (Table 1) - there may be other clinically important comorbidities among patients undergoing endovascular stroke treatment that could contribute to clinical outcomes, such as pre-existing dementia or other medical conditions not recorded in these trials. The THRIVE score will require confirmation as a predictive tool by using a separate endovascular stroke-treatment cohort for validation. If the THRIVE score is validated, it will be important to explore in other datasets the relationships between the THRIVE score and other possible predictive factors, such as ASPECTS, CT or MR perfusion data, and admission temperature. It will also be important to test the generalizability of the THRIVE score to patients undergoing other types of stroke treatment such as intravenous $\mathrm{PAA}$, intra-arterial thrombolysis, ${ }^{1,11}$ or use of the Penumbra System (Penumbra, Alameda, California) clot aspiration catheter. ${ }^{12}$

Other approaches to predict outcome among patients undergoing endovascular stroke treatment have been explored. For example, analysis of early ischemic changes on admission noncontrast head CT by using the ASPECTS predicted outcome among patients in the PROACT-II study of intra-arterial prourokinase. ${ }^{13}$ A 3-point score encompassing age, NIHSS, and admission hyperglycemia that predicts poor outcome by the time of hospital discharge was recently developed and validated. ${ }^{9}$ Of the 3 variables included in this score, age and NIHSS are included in the THRIVE score, and admission hyperglycemia was found to have an effect in the CDS similar to 
A

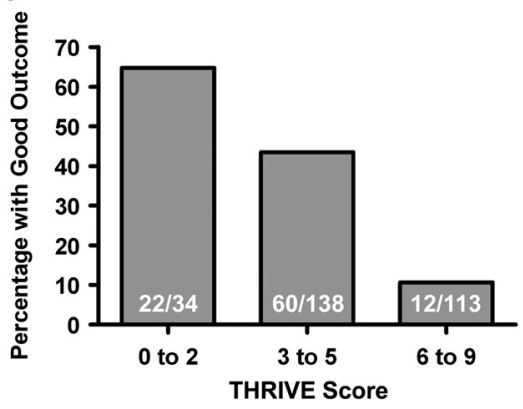

B

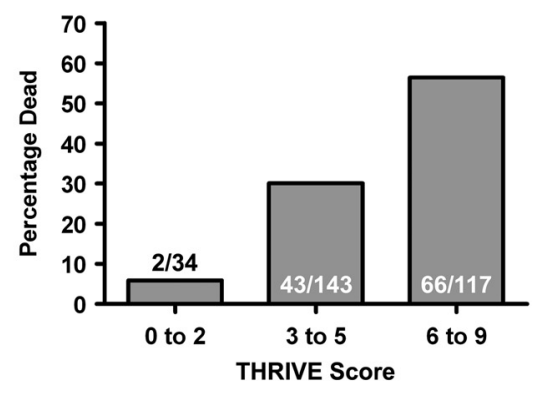

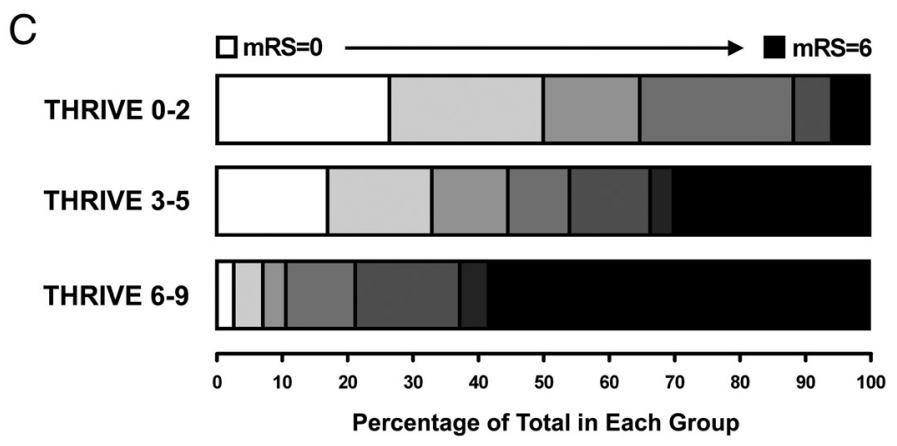

Fig 2. Association of the THRIVE score with outcome. A, Association of the 3 strata of the THRIVE score (0-2, 3-5, and 6-9) with good outcome (mRS $0-2$ at 90 days). B, Association of the 3 strata of the THRIVE score $(0-2,3-5$, and $6-9)$ with death by 90 days. $C$, Range of outcomes measured by the mRS at 90 days, according to the THRIVE score. For each of the 3 strata of the THRIVE score ( $0-2$ versus $3-5$ versus $6-9)$, the range of outcomes on the 90-day mRS is shown. Each increased level of the mRS is displayed by progressively darker gray-scale values, from white $(\mathrm{mRS}=0)$ to black $(\mathrm{mRS}=6)$. There were no patients with $\mathrm{mRS}=5$ in the THRIVE score $0-2$ group.

DM. It remains to be determined whether imaging data (such as ASPECTS or data from advanced neuroimaging techniques to visualize territory at risk such as MR perfusion or CT perfusion) might enhance the predictive power of the THRIVE score.

\section{Conclusions}

Age, NIHSS, and specific chronic diseases (HTN, DM, and AFib) contribute important information to the prediction of outcome after endovascular stroke treatment. The THRIVE score, a simple-to-use score that incorporates age, NIHSS, and chronic disease burden, may be useful in comparing patients across endovascular stroke treatment cohorts and in clinical trials. With further validation and potential use in the context of trials, the THRIVE score may become useful as a tool to enhance patient selection for endovascular stroke treatment. A THRIVE score calculator with associated outcomes can be accessed online. ${ }^{14}$

\section{Acknowledgments}

We thank Cindy Jahans, Gary Walker, and Maria Sainz of Concentric Medical, Mountain View, California, for supplying us with data from the MERCI and Multi MERCI trials. The authors would also like to thank Carol Conell, PhD, of the Kaiser Permanente Division of Research for her critical review of the manuscript and helpful statistical suggestions.

\section{References}

1. Furlan A, Higashida R, Wechsler L, et al. Intra-arterial prourokinase for acute ischemic stroke: The PROACT II study—a randomized controlled trial. Prolyse in Acute Cerebral Thromboembolism. JAMA 1999;282:2003-11
2. Smith WS, Sung G, Saver J, et al. Mechanical thrombectomy for acute ischemic stroke: final results of the Multi MERCI trial. Stroke 2008;39:1205-12

3. Smith WS, Sung G, Starkman S, et al. Safety and efficacy of mechanical embolectomy in acute ischemic stroke: results of the MERCI trial. Stroke 2005;36:1432-38

4. Smith WS. Safety of mechanical thrombectomy and intravenous tissue plasminogen activator in acute ischemic stroke: results of the multi Mechanical Embolus Removal in Cerebral Ischemia (MERCI) trial, part I. AJNR Am J Neuroradiol 2006;27:1177-82

5. Flint AC, Duckwiler GR, Budzik RF, et al. Mechanical thrombectomy of intracranial internal carotid occlusion: pooled results of the MERCI and Multi MERCI Part I trials. Stroke 2007;38:1274-80. Epub 2007 Mar 1

6. Lutsep HL, Rymer MM, Nesbit GM. Vertebrobasilar revascularization rates and outcomes in the MERCI and multi-MERCI trials. J Stroke Cerebrovasc Dis 2008; $17: 55-57$

7. Josephson SA, Saver JL, Smith WS. Comparison of mechanical embolectomy and intraarterial thrombolysis in acute ischemic stroke within the MCA: MERCI and Multi MERCI compared to PROACT II. Neurocrit Care 2009; 10:43-49

8. Williams DO, Borer J, Braunwald E, et al. Intravenous recombinant tissue-type plasminogen activator in patients with acute myocardial infarction: a report from the NHLBI Thrombolysis in Myocardial Infarction trial. Circulation 1986;73:338-46

9. Hallevi $\mathrm{H}$, Barreto $\mathrm{AD}$, Liebeskind $\mathrm{DS}$, et al. Identifying patients at high risk for poor outcome after intra-arterial therapy for acute ischemic stroke. Stroke 2009;40:1780-85

10. Flint AC, Smith WS. Predicting long-term outcomes for ischemic stroke based on admission variables. Stroke Rounds 2008;2:1-6

11. del Zoppo GJ, Higashida RT, Furlan AJ, et al. PROACT: a phase II randomized trial of recombinant pro-urokinase by direct arterial delivery in acute middle cerebral artery stroke-PROACT Investigators. Prolyse in Acute Cerebral Thromboembolism. Stroke 1998;29:4-11

12. Bose A, Henkes H, Alfke K, et al. The Penumbra System: a mechanical device for the treatment of acute stroke due to thromboembolism. AJNR Am J Neuroradiol 2008;29:1409-13. Epub 2008 May 22

13. Hill MD, Rowley HA, Adler F, et al. Selection of acute ischemic stroke patients for intra-arterial thrombolysis with pro-urokinase by using ASPECTS. Stroke 2003;34:1925-31

14. THRIVE Score Calculator. Available at: http://www.thrivescore.org. Accessed March 9, 2010. 